\title{
Theranostics
}

Editorial

2011; 1:28-29

\section{Integrin Targeted Imaging and Therapy}

\section{Xiaoyuan Chen $\bowtie$}

Laboratory of Molecular Imaging and Nanomedicine, National Institute of Biomedical Imaging and Bioengineering, National Institutes of Health, USA

Corresponding author: shawn.chen@nih.gov

() Ivyspring International Publisher. This is an open-access article distributed under the terms of the Creative Commons License (http://creativecommons.org/ licenses/by-nc-nd/3.0/). Reproduction is permitted for personal, noncommercial use, provided that the article is in whole, unmodified, and properly cited.

Published: 2011.01.12

\begin{abstract}
This theme issue provides an overview on the biology and pathology of various integrins as well as in-depth discussion on the use of integrin as targeting molecules for molecular imaging and molecular therapy.
\end{abstract}

Key words: Integrin, molecular imaging, molecular therapy, theranostics

Integrins are a family of heaterodimeric transmembrane receptors each consisting of an $\alpha$ and a $\beta$ subunit. A total of $18 \alpha$ and $8 \beta$ subunits are known in mammalian cells, forming at least 24 different integrin receptors. Each integrin subunit contains a large extracellular domain, a single transmembrane domain and a short cytoplasmic domain. The composition and morphology of integrin-dependent adhesions vary with the cell type, matrix and integrin, contributing to cell advancement, tissue remodeling and repair. Based on the key roles they play in angiogenesis, leukocytes function and tumor development and easy accessibility as cell surface receptors interacting with extracellular ligands, the integrin superfamily, integrin $a v \beta 3$ in particular, have been extensively investigated as imaging and therapy targets. This theme collection of articles first introduces integrin biology followed by integrin targeted imaging and therapy applications. Almost every imaging modality, including positron emission tomography (PET), single photon emission computed tomography (SPECT), optical, magnetic resonance (MR), ultrasound, and the combination of multimodality imaging has been tried to visualize and quantify integrin expression for lesion detection and for therapy response monitoring.
As for therapy applications, integrin antagonists can serve as antiangiogenic drugs, integrin ligands can also act as delivery vehicles for chemo, radio and gene therapeutics. This theme issue consists of 12 reviews and was contributed by 28 authors worldwide who are among the world's prominent scientists in molecular imaging and/or molecular pharmaceutics.

Niu and Chen [1] start with an overall description of the structure, expression pattern, and biologi$\mathrm{cal} /$ pathological function of various integrins. Beer at al. [2] describe different strategies of labeling integrin av $\beta 3$ ligands for PET imaging of tumor angiogenesis and metastasis in both preclinical models and clinical patients. Zhou et al. [3] focus on recent developments of integrin av $\beta 3$ targeted radiotracers for SPECT imaging of tumors and integrin aIIb $\beta 3$ targeted radiotracers for SPECT imaging of thrombosis. Approaches to maximize targeting capability of cyclic RGD peptides and to improve the radiotracer excretion kinetics are also discussed. Ye and Chen [4] summarize fluorescent dyes and quantum dots labeled peptides for targeting integrin av $\beta 3$ and several other integrin subtypes such as $\alpha 4 \beta 1, \alpha 3$, and $\beta 3$. Integrin-targeted activatable optical probes are also briefly mentioned. Tan and Lu [5] overview a number of efficient deliv- 
ery systems for loading sufficient amount of Gd(III) chelates or superparamagnetic nanoparticles for $\mathrm{T} 1$ and T2-weighted MR imaging of integrin expression in animal models. Kiessling et al. [6] give concrete examples on the use of molecular ultrasound to characterize integrin expression on blood vessels. Integrin targeted microbubbles can be applied in oncology to identify and characterize tumor angiogenesis and to assess tumor response to antiangiogenic drugs as well as to radiotherapy; it can also be a valuable tool for the characterization of a broad spectrum of diseases. Zhang et al. [7] argue that combination of certain modalities can offer synergistic advantages of over single modality and concentrates on integrin targeting contrast agents that can be detected by two or more imaging modalities, most of which are based on nanoparticle platforms. Morrison and Cuthbertson [8] emphasize the importance of radiolabeled RGD peptides with favorable in vivo kinetics and tumor targeting properties to quantify changes in tumor vascularity following anti-cancer therapies, particularly those that predominantly target tumor blood vessels. Millard et al. [9] give rationales on how to design and develop potent, selective inhibitors for a number of integrins involved in platelet aggregation, inflammatory responses, angiongenesis, neovascularization and tumor growth, based on the advances in structural characterization of integrin-ligand interactions. Chen and Chen [10] summarize the bioconjugation chemistry of coupling integrin ligands and chemotherapeutics to increase the drug load in the tumor area while sparing the normal tissue from the exposure to the cytotoxic chemicals. Liu et al. [11] illustrates the potential of beta-emitter labeled antibodies and peptides for internal radiotherapy of integrin positive tumors. Finally, Juliano et al. [12] review recent work concerning the targeted delivery of antisense and siRNA oligonuclides via integrins and consequently enhance the pharmacological efficacy of therapeutic oligonucleotides.

In summary, it is a very timely collection of literature reports and provides an overview on the bi- ology and pathology of various integrins as well as in-depth discussion on the use of integrin targeting molecules as probes for various imaging modalities: PET, SPECT, optical imaging, MRI and ultrasound imaging. In addition, this issue also discusses the use of these targeting ligands to deliver radionuclides, nanoparticles, chemotherapeutics and therapeutic nucleic acids such as antisense and siRNA oligonucleotides to disease sites.

I thank all the authors for writing this important theme issue, which will undoubtedly benefit many experts and students in the fields of cancer theranostics.

\section{Conflict of Interest}

The author has declared that no conflict of interest exists.

\section{References}

1. Niu $G$, Chen $X$. Why integrin as a primary target for imaging and therapy. Theranostics 2011; 1: 30-47.

2. Beer AJ, Kessler H, Wester HJ, Schwaiger M. PET imaging of integrin $\alpha V \beta 3$ expression. Theranostics 2011; 1:48-57.

3. Zhou Y, Chakraborty S, Liu S. Radiolabeled cyclic RGD peptides as radiotracers for imaging tumors and thrombosis by SPECT. Theranostics 2011; 1: 58-82.

4. Ye $Y$, Chen $X$. Integrin targeting for tumor optical imaging. Theranostics 2011; 1: 102-126.

5. Tan M, Lu Z-R. Integrin targeted MR imaging. Theranostics 2011; 1: 83-101.

6. Kiessling F, Gaetjens J, Palmowski m. Application of molecular ultrasound for imaging integrin expression. Theranostics 2011; 1: 127-134.

7. Zhang Y, Yang Y, Cai W. Multimodality imaging of integrin av $\beta 3$ expression. Theranostics 2011; 1: 135-148.

8. Morrison $\mathrm{M}$, Cuthbertson A. Integrin imaging to evaluate treatment response. Theranostics 2011; 1: 149-153.

9. Millard M, Odde S, Neamati N. Integrin targeted therapeutics. Theranostics 2011; 1:154-188.

10. Chen K, Chen X. Integrin targeted delivery of chemotherapeutics. Theranostics 2011; 1:189-200.

11. Liu Z, Wang F, Chen X. Integrin targeted delivery of radiotherapeutics. Theranostics 2011; 1: 201-210.

12. Juliano RL, Ming $X$, Nakagawa $O, X u$ R, Yoo H. Integrin targeted delivery of gene therapeutics. Theranostics 2011; 1 : 211-219. 